\title{
Especificidad contextual diferencial en la habituación de las respuestas de parpadeo y aceleración cardiaca en humanos*
}

\author{
Differential Context-Specificity in the Habituation of \\ the Eyeblink and Cardiac Responses in Humans
}

Recibido: diciembre 12 de 2012 | Revisado: mayo 29 de 2014 | Aceptado: mayo 29 de 2014

\author{
JoRge A. PINTO ** \\ Sebastián A. Becerra \\ FERNANDO P. PONCE \\ EDGar H. VOGEL \\ Universidad de Talca, Maule, Chile
}

doi:10.11144/Javeriana.UPSY13-4.ecdh

Para citar este artículo: Pinto, J. A., Becerra, S. A., Ponce, F. P., \& Vogel, E. H. (2014). Especificidad contextual diferencial en la habituación de las respuestas de parpadeo y aceleración cardiaca en humanos. Universitas Psychologica, 13(4), 12045-12054. http://dx.doi.org/10.11144/Javeriana. UPSY13-4.ecdh

* Agradecimientos: El presente estudio fue financiado por el proyecto Fondecyt $\mathrm{N}^{\circ} 1120265$ a Edgar Vogel y por la Universidad de Talca a través del programa de investigación "Calidad de Vida y Ambientes Saludables" (Res. 387/2007).

** Correos electrónicos: jpinto@alumnos.utalca.cl, sbecerra@alumnos.utalca.cl, fponce@utalca.cl, evogel@utalca.cl

\section{RES UMEN}

Wagner (1978) propuso que la habituación, definida como una disminución en la respuesta a un estímulo que se repite, dependería de la formación de una asociación entre el contexto y el estímulo. Según este enfoque, la habituación debería ser contexto-específica, es decir, la respuesta habituada en un contexto debería deshabituarse al presentar el estímulo en un contexto novedoso. Esta hipótesis fue examinada a través de un experimento donde se sometió a un grupo de estudiantes a una sesión de habituación consistente en 60 repeticiones de un estímulo provocador de reacciones de parpadeo y aceleración cardiaca. Posteriormente, en una sesión de prueba se midió la amplitud de estas respuestas, presentando el estímulo en el mismo contexto (Grupo Igual) o en un contexto distinto a aquel donde ocurrió la habituación (Grupo Diferente).

Los resultados de la prueba arrojaron evidencia de especificidad contextual diferencial para ambas respuestas, ya que la respuesta de aceleración cardiaca resultó disminuida en el grupo igual pero no en el grupo diferente (revelando especificidad), mientras que la respuesta de parpadeo estuvo igualmente disminuida en ambos grupos (revelando ausencia de especificidad). Estos hallazgos confirman observaciones previas con ratas que demuestran que el control contextual de la habituación depende de la naturaleza de la respuesta.

Palabras clave

habituación; aprendizaje contextual; especificidad contextual

\begin{abstract}
A B S T R A C T
Wagner (1978) proposed that habituation, defined as a decrease in responding to a repeated stimulus, would depend on the formation of an association between the stimulus and the context. According to this approach, habituation should be context-specific; that is, a response that was habituated in a given context should dishabituate when the stimulus is presented in a novel context. This hypothesis was examined in an experiment in which a group of students received a habituation session consisting of 60 repetitions of a stimulus capable of evoking eyeblink and heart-rate acceleration reactions. Subsequently, in a testing session the amplitude of these responses was examined by presenting the stimulus in the same context used in the habituation session (Group Same) or in an alternative context (Group Different). The
\end{abstract}


results provided evidence of differential context-specificity for the two responses, since the heart-rate acceleration response was diminished in the group same but not in the group different (revealing specificity), while the eyeblink response was diminished in both groups (revealing no specificity). These findings are consistent with previous observations in rats demonstrating that the contextual control of habituation depends on the nature of the measured response.

Keywords

habituation; context learning; context-specificity

\section{Introducción}

La repetición de un estímulo normalmente desencadena una disminución progresiva en la frecuencia o amplitud de la respuesta provocada por este. En aquellas ocasiones en que esta disminución no es causada por fatiga muscular ni adaptación sensorial, se concluye que ha ocurrido una de las formas más básicas de aprendizaje, denominada habituación. Si el decremento en la repuesta es duradero, se habla de habituación de largo plazo, mientras que si es transitorio, se habla de habituación de corto plazo (Vogel \& Wagner, 2005).

Se ha reportado evidencia de habituación en especies tan diversas como protozoos, peces, moluscos, ratas, conejos, gatos, perros y humanos (Davis, 1970; Dycus \& Powers, 1997; Frost, Brandon, \& van Zyl, 2006; Peeke \& Veno, 1973; Seal \& Zbrozyna, 1978; Thompson \& Spencer, 1966; Whitlow, 1975; Wood, 1973). La abundante información acumulada durante más de un siglo de investigación experimental ha permitido identificar un conjunto de características o parámetros de la habituación, cuyas principales regularidades empíricas han permanecido relativamente indiscutidas, desde que fueran enunciadas sistemáticamente por Thompson y Spencer (1966).

Sin embargo, un fenómeno que ha estado sometido a considerable debate teórico y empírico se refiere a la posible especificidad contextual de la habituación. Según las teorías convencionales, la habituación es un fenómeno de aprendizaje no asociativo que depende únicamente de la repetición del estímulo. La noción más simple es aquella planteada por Groves y Thompson (1970), quienes sugirieron que la habituación es el resultado de un declive paulatino en la eficacia de la transmisión de la señal en el circuito estímulo-respuesta. Según este enfoque y otros similares (p. ej., Castellucci, Pinsker, Kupfermann, \& Kandel, 1970; Hall, 1991; Sokolov, 1960), la habituación se debería a influencias diminutivas intrínsecas que ocurren en el circuito estímulo-respuesta en cuestión y que como tales dependerían únicamente de la repetición del estímulo y no se verían influidas por el contexto en el cual ocurren.

Alternativamente, Wagner y sus colaboradores (Wagner, 1976, 1978, 1979, 1981; Wagner \& Vogel, 2010; Whitlow \& Wagner, 1984) sostienen que la habituación, al menos en algunos sistemas de respuesta, es el resultado de influencias extrínsecas al circuito estímulo-respuesta. Estas influencias serían de carácter asociativo y provendrían principalmente de la ocurrencia del condicionamiento Pavloviano entre el contexto y el estímulo. De acuerdo a este enfoque, un estímulo que se repite en un determinado contexto termina por ser anticipado o esperado en ese contexto $y$, por lo tanto, resulta menos efectivo en producir su respuesta. De este modo, una respuesta habituada en un contexto debería mostrar una significativa recuperación al presentar el estímulo en otro contexto, en el cual no se había presentado con anterioridad.

Jordan, Strasser y McHale (2000) publicaron evidencia problemática tanto para los enfoques asociativos como no asociativos, al demostrar que para un mismo estímulo, la habituación de una de sus respuestas sufriría de un decremento que es específico del contexto, mientras que para otras respuestas no existiría tal especificidad. Para ello, evaluaron simultáneamente las conductas de supresión de consumo de agua y de sobresalto producidas por un estímulo auditivo intenso en ratas. Pese a que se demostró la existencia de habituación de ambas respuestas, solo la respuesta de supresión mostró una importante recuperación cuando el estímulo fue probado en un contexto distinto de aquel en el cual ocurrió la habituación (Experimentos 1 y 2).

El hecho de que Jordan et al. (2000) hayan demostrado simultáneamente ausencia y presencia de control contextual de distintas respuestas ante el mismo estímulo, sugiere que podrían existir dis- 
tintos mecanismos de habituación para distintos sistemas de respuesta. Estudios previos realizados con un solo sistema de respuesta apuntaban en la misma dirección. Por ejemplo, al menos 2 estudios han demostrado que la habituación de la respuesta de sobresalto se transfiere completamente de un contexto a otro (Borszcz, Cranney, \& Leaton, 1989; Marlin \& Miller, 1981). Por otra parte, el control contextual de la habituación de la respuesta de supresión fue confirmado por Jordan et al. (2000) en experimentos independientes (Experimentos 3, 4 y 5), y otros estudios han encontrado evidencia de especificidad contextual con procedimientos tales como las respuestas de escape en cangrejos (Tomsic, Pedreira, Hermitte, Romano, \& Maldonado, 1998), de movimiento en invertebrados (Rankin, 2000) y de orientación en ratas (Hall y Channel, 1985; Jordan et al., 2000 [Experimento 5]).

La excepcionalidad de la repuesta de sobresalto en no mostrar control contextual de la habituación sugiere que en este caso podría operar un mecanismo de habituación diferente a aquel que opera en otras respuestas. Esto es consistente con evidencia que señala que el curso de la habituación de las respuestas de sobresalto no se correlaciona significativamente con la habituación de la respuesta de supresión, y que lesiones en la formación reticular afectan severamente la habituación de la respuesta de sobresalto, sin afectar la habituación de la respuesta de supresión (Jordan \& Leaton, 1982).

Otra particularidad de la respuesta de sobresalto se refiere a su rapidísimo curso temporal en comparación con otras repuestas. El sobresalto se inicia aproximadamente $10 \mathrm{~ms}$ después de presentado el estímulo y alcanza su amplitud máxima aproximadamente a los $50 \mathrm{~ms}$. Por el contrario, aquellas respuestas donde se ha manifestado control contextual, tales como la supresión, escape y orientación, el período de medición de las respuestas se extiende por varios segundos. Según el análisis teórico realizado por Wagner y Vogel (2010), respuestas de curso temporal rápido tendrían menos posibilidades de asociarse con estímulos contextuales.

Si efectivamente el curso temporal de la respuesta tiene un impacto en el grado de control contextual de la habituación, esto debería observarse con otros sistemas de respuesta. Para ello, en este artículo se describe los resultados de un experimento en el cual se examinó la posible especificidad contextual de dos respuestas, parpadeo y aceleración cardiaca en humanos, en los cuales se ha demostrado la ocurrencia de habituación (p. ej., Grayson, 1982; Ponce, Quintana, Philominraj, \& Vogel, 2011). Estas respuestas pueden ser elicitadas tanto por un estímulo auditivo de alta intensidad o bien por un leve soplido de aire en el ojo. El parpadeo es una respuesta cuyo curso temporal es semejante a la respuesta de sobresalto, desarrollándose completamente en menos de 1 segundo, mientras que la aceleración cardiaca se despliega en un intervalo de aproximadamente 60 segundos. De este modo, se espera que la respuesta de parpadeo tenga menos probabilidades de asociarse con el contexto y así, por lo tanto, exhibir un menor grado de especificidad contextual que la respuesta de aceleración cardiaca.

\section{Método}

\section{Participantes}

Un total de 20 estudiantes de la Universidad de Talca, sin experiencia previa en este tipo de estudios, participaron voluntariamente en el experimento. Sus edades fluctuaron entre los 19 y 24 años. Los participantes firmaron un consentimiento informado con la opción de renunciar a participar del experimento en el momento en que lo consideraran pertinente. Los participantes del estudio fueron asignados a uno de los dos grupos experimentales (Igual y Diferente).

El contenido del consentimiento informado y el procedimiento del experimento fueron aprobados por el Comité de Ética de la Universidad de Talca y posteriormente por el Comité de Ética de CO. NICYT, al asignar el financiamiento del proyecto regular Fondecyt $\mathrm{N}^{\circ} 1120265$, al cual se adscribe la presente investigación.

\section{Aparatos}

El experimento fue conducido en dos contextos marcadamente distintos. El contexto "A" fue una 
habitación iluminada con una luz tenue de color amarillo, de $250 \mathrm{~cm}$ ancho $\times 270 \mathrm{~cm}$ largo x $250 \mathrm{~cm}$ alto, la cual fue arreglada como un ambiente de sala de estar con un sillón amarillo, dos cuadros, una mesa con libros y adornos, dos plantas, una mesa con una lámpara pequeña, una lámpara de pie y un perchero. El contexto "B" fue una habitación iluminada con una luz de color blanco, de $250 \mathrm{~cm}$ ancho x $510 \mathrm{~cm}$ largo x $250 \mathrm{~cm}$ alto, la cual fue arreglada como un ambiente de laboratorio, con un sillón azul, dos paneles de equipos experimentales, cuatro escritorios aislados por paneles con un computador y un afiche de información científica. Los participantes ingresaron a uno de estos contextos y se sentaron en un sillón, donde se realizaron mediciones simultáneas de las respuestas de parpadeo y aceleración cardíaca durante las fases del experimento.

El sistema Eyeblink Conditioning System (San Diego Instruments Inc., San Diego, CA) fue utilizado para configurar la sesión de entrenamiento, la administración de los estímulos y la medición de la amplitud de las respuestas de parpadeo y aceleración cardiaca.

Los participantes del estudio recibieron un estímulo táctil o un estímulo auditivo, ambos capaces de provocar respuestas de parpadeo y aceleración cardiaca. El estímulo táctil consistió en un soplo de aire en el contorno del ojo ( 9 psi, $500 \mathrm{~ms})$, administrado a través de un tubo de aire sujetado por un cintillo. El estímulo auditivo consistió en un tono $(90 \mathrm{~dB}, 500 \mathrm{~ms})$, administrado a través de audífonos MAICO.

La respuesta palpebral fue medida a través de un sensor infrarrojo colocado frente al ojo derecho de los participantes y sujetado por un cintillo que permitía mantener fija la posición del sensor, independiente de los cambios posturales. La respuesta de parpadeo produce una interrupción en la emisión infrarroja, la que se traduce en cambios en el voltaje transmitido por el sensor. Se registró el valor de voltaje con una frecuencia de 1 muestra por cada $10 \mathrm{~ms}$ durante $100.000 \mathrm{~ms}$. Durante los 200 ms que anteceden a la presentación del estímulo, se registraron los voltajes en ausencia de estimulación (línea base), luego de lo cual fue presentado el estímulo por $500 \mathrm{~ms}$. El voltaje máximo obtenido durante los primeros $200 \mathrm{~ms}$ de la duración del estímulo fue considerado como la amplitud de la respuesta de parpadeo. Una respuesta de parpadeo fue considerada como válida si el registro indicaba una línea base inferior al 20\% del valor de voltaje promedio obtenido en ausencia de estimulación.

La repuesta de aceleración cardiaca fue medida a través de un sensor infrarrojo ubicado en el dedo índice izquierdo de los participantes. El sensor capta cambios en el volumen sanguíneo producido por el pulso cardiaco, lo cual se traduce en señales eléctricas registradas en el computador. Se tomó una muestra por cada $10 \mathrm{~ms}$, durante $100.000 \mathrm{~ms}$. El tiempo transcurrido entre 2 pulsos permitió calcular la frecuencia cardiaca que ocurre segundo a segundo. El promedio de la frecuencia cardiaca durante los 15 segundos previos a la estimulación se utilizó como línea base. La respuesta de aceleración cardiaca producida por la estimulación se estimó como la diferencia entre la línea base y la frecuencia cardiaca segundo a segundo, durante los 80 segundos que siguen a la presentación del estímulo.

\section{Procedimiento}

El experimento contempló cuatro fases: pre-exposición, pre-prueba, entrenamiento y post-prueba. En la fase de pre-exposición los participantes fueron expuestos a uno de los dos contextos durante 10 min, sin recibir ningún estímulo (la mitad de los participantes fueron expuestos al contexto A y la otra mitad al contexto B). El objetivo de esta fase fue que los participantes se familiarizaran con el contexto de prueba. Al cumplirse el tiempo, abandonaron la habitación para permanecer en una sala de espera durante $5 \mathrm{~min}$. Posteriormente, en la fase de pre-prueba, ingresaron a uno de los contextos experimentales donde fueron expuestos a 4 ensayos con uno de los estímulos provocadores de respuestas (11 participantes recibieron un estímulo auditivo y 9, uno táctil) con un intervalo entre estímulos de 100 s. Luego, en la fase de entrenamiento, se presentaron 60 ensayos con el mismo estímulo a intervalos de $30 \mathrm{~s}$. Al finalizar este entrenamiento, los participantes nuevamente abandonaron la ha- 
bitación durante $5 \mathrm{~min}$, luego de lo cual realizaron la fase de post-prueba, que consistió en 4 presentaciones del estímulo habituado a un intervalo de 100 s. Para los sujetos del Grupo Igual todas las fases del experimento ocurrieron en el mismo contexto, mientras que para los del Grupo Diferente la fase de pre-prueba y entrenamiento ocurrieron en un contexto distinto al de la fase de post-prueba.

Se esperaría que de existir especificidad contextual de la habituación para cada una de las respuestas, los participantes que fueron sometidos a una post-prueba en un contexto distinto de aquel en el cual fueron habituados, deberían mostrar una significativa recuperación en los niveles de respuesta al estímulo habituado, en comparación con aquellos que fueron habituados y sometidos a una post-prueba en el mismo contexto.

\section{Análisis estadísticos}

Con el fin de reducir la variabilidad en las puntuaciones producidas por el hecho que cada participante presenta niveles basales de respuesta distintos, se calculó un índice de decremento para cada sujeto, dividiendo su amplitud promedio de respuesta en la post-prueba por la suma de la amplitud promedio en la post-prueba más la amplitud promedio en la pre-prueba. De este modo, el nivel de respuesta en la post-prueba se expresa como una proporción del nivel global de respuesta de cada participante comparado consigo mismo. Si la respuesta en la post-prueba es idéntica a la pre-prueba, el índice de decremento es igual a 0.5 , lo cual indica ausencia de decremento o habituación nula. Por otra parte, si el índice de decremento es inferior a 0.5 , esto significa que se responde menos en la post-prueba que en la pre-prueba y que, por lo tanto, ha ocurrido habituación.

Con el fin de evitar los valores negativos que se podrían obtener en algunos ensayos con respuestas cardiacas inferiores a la línea base (desaceleración), lo cual haría perder significado al índice de decremento, se hizo una transformación lineal de los puntajes de cada participante igualando su valor mínimo a cero.

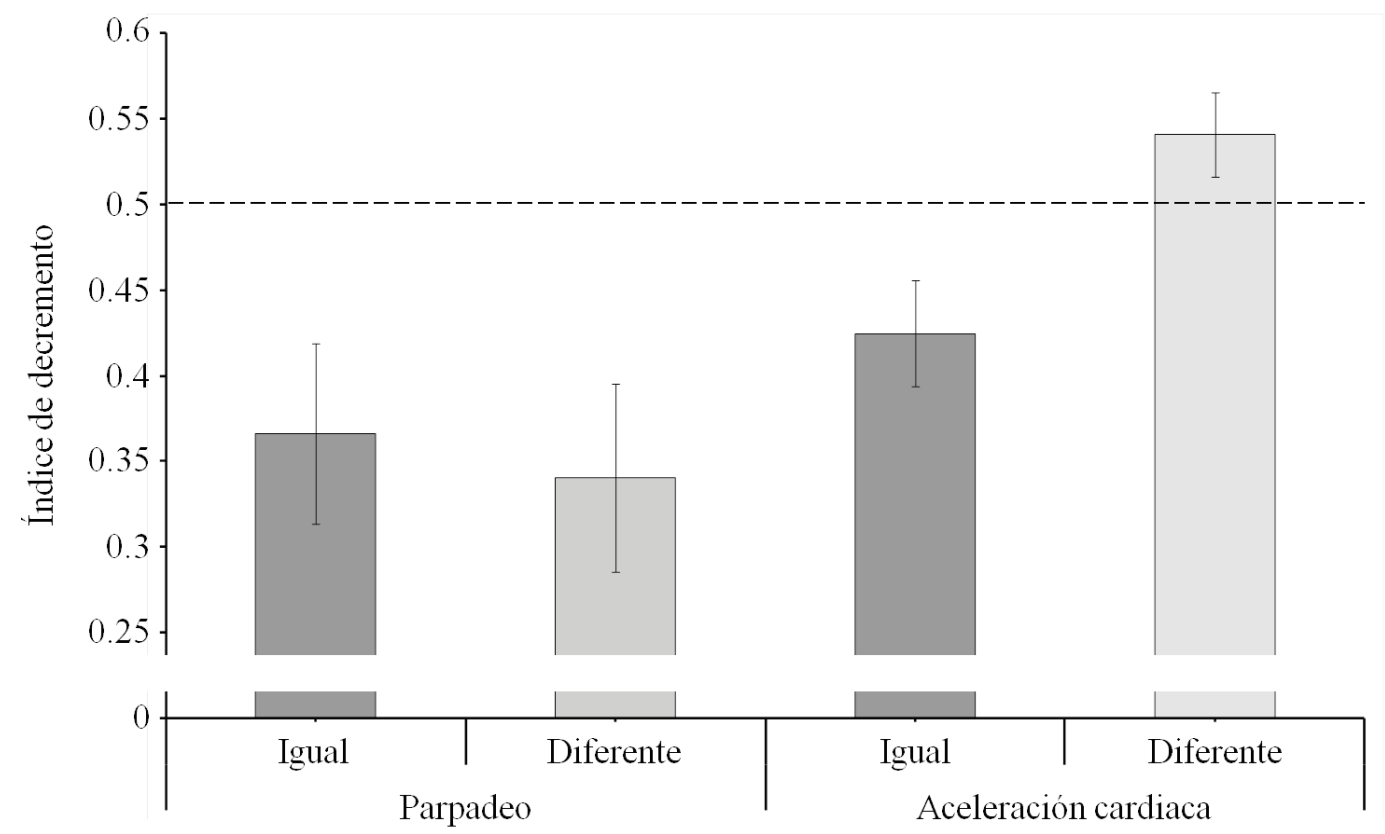

Figura 1. Índice de decremento promedio de las respuestas de parpadeo y aceleración cardiaca para los grupos Igual y Diferente. Las barras de error representan el error estándar de la media.

Fuente: elaboración propia 
Para evaluar la significancia estadística de los efectos del experimento, se realizaron dos tipos de análisis. En primer lugar, con el fin de detectar la presencia de habituación en cada sistema de respuesta en ambos grupos, se examinó si el índice de decremento difiere significativamente de 0.5 a través de una prueba $t$ de Student de una muestra. En segundo lugar, con el fin de comparar el índice de decremento del Grupo Igual con el Grupo Diferente, se realizó una prueba $t$ para muestras independientes para cada sistema de respuesta.

\section{Resultados}

La Figura 1 presenta los índices de decremento para ambos grupos, tanto para la respuesta de parpadeo como de aceleración cardiaca. La línea punteada sirve como referencia para examinar la presencia de habituación en cada grupo y sistema de respuesta. Los valores por debajo de la línea indican que las respuestas en la post-prueba están disminuidas en comparación con la pre-prueba (habituación).

Como se puede observar en la Figura 1, en el caso de la respuesta palpebral los índices de decremento son sustancialmente inferiores a 0.5 , en ambos grupos, lo cual indica que la manipulación fue exitosa en lograr habituación, y que esta se manifiesta de la misma manera independientemente de si el contexto de la post-prueba es el mismo que en la fase de habituación (Grupo Igual) o en un contexto distinto (Grupo Diferente). Los análisis estadísticos confirman esta ausencia de control contextual de la habituación, ya que el índice de decremento es significativamente inferior a 0.5 tanto en el Grupo Igual $(t(9)=-2.541, \mathrm{p}=0.032)$ como en el Grupo Diferente $(t(9)=-2.925, p=0.017)$. Más aún, no se observaron diferencias significativas entre los índices de decremento de ambos grupos $(t(18)=$ $-0.339 ; p=0.738$ ), lo cual indica que experimentaron niveles similares de habituación.

Por otra parte, la Figura 1 indica que los valores obtenidos con la respuesta de aceleración cardiaca sugieren la existencia de especificidad contextual en la forma de una recuperación en el nivel de respuesta del grupo diferente en la post-prueba. Esto se confirma estadísticamente, por cuanto el índice de decremento es significativamente inferior a 0.5 en el Grupo Igual $(t(9)=-2.447, p=0.037)$, situación que no ocurre en el Grupo Diferente $(t(9)=1.658$, $p=0.132)$. Asimismo, el índice de decremento del Grupo Igual fue significativamente inferior al del Grupo Diferente $(t(18)=2.9547, p=0.009)$.

Estos datos demuestran que la habituación de la respuesta palpebral se transfiere completamente desde el contexto de habituación al contexto de prueba, en el presente experimento. Por el contrario, en el caso de la respuesta de aceleración cardiaca se observó una recuperación virtualmente completa de la respuesta, cuando el estímulo fue presentado en un contexto diferente a aquel donde ocurrió la habituación.

\section{Discusión}

Los resultados de esta investigación arrojan evidencia de control contextual diferencial de la habituación de 2 respuestas elicitadas por el mismo estímulo, en humanos. Adicionalmente, la habituación de la repuesta de aceleración cardiaca no se transfiere desde el contexto de habituación al contexto de prueba, indicando especificidad contextual del decremento. Por otra parte, no se observa tal control contextual en la habituación de la respuesta palpebral, observándose una transferencia aparentemente total de la habituación, desde el contexto original hacia uno novedoso. Estos hallazgos extienden las observaciones de Jordan et al. (2000), quienes demostraron control contextual de la habituación de las repuestas de supresión y orientación, pero no de sobresalto en ratas.

El procedimiento seguido por Jordan et al. (2000) y por la presente investigación, en el cual se examina simultáneamente el curso de la habituación de más de una respuesta, permite descartar explicaciones alternativas con las cuales se podrían cuestionar los resultados de otros estudios, donde se examina un solo sistema de respuesta en forma aislada. Por ejemplo, en aquellos estudios donde se demuestra ausencia de control contextual de una respuesta (p. ej., Marlin \& Miller, 1981), es posible argüir que dicha ausencia se debe a la generalización entre contextos y no a la falta de asociación 
entre el contexto y el estímulo. Esta hipótesis pierde sustento con los datos de la presente investigación y aquellos de Jordan et al. (2000), puesto que de existir suficiente generalización entre los contextos, esto debería haber afectado igualmente a todas las respuestas, lo cual no ocurrió.

Alternativamente, en aquellos estudios donde se ha demostrado especificidad contextual con un solo sistema de respuesta (p. ej., Evans \& Hammond, 1983; Hall \& Channel, 1985; Kruse, Strippling, \& Clayton, 2004; Rankin, 2000; Shalter, Fentress, \& Young, 1977), podría conjeturarse que la recuperación en la respuesta que ocurre al cambiar el contexto se debe a una suerte de sensibilización causada por la novedad del contexto de prueba y no a pérdida de habituación. Si bien algunos estudios han intentado reducir este efecto pre-exponiendo a los animales al contexto de prueba antes de ejecutar la sesión de habituación, siempre es posible argüir que esta familiarización con el contexto no fue suficiente para reducir su poder sensibilizador. Sin embargo, esta hipótesis pierde plausibilidad cuando se consideran los datos de la presente investigación, puesto que de existir sensibilización provocada por el contexto novedoso, esta debería afectar a ambos sistemas de respuesta y no solo a la repuesta cardiaca.

La ausencia de control contextual de la respuesta de sobresalto en ratas reportada por Jordan et al. (2000) y de parpadeo en humanos reportada en el presente artículo, podría significar que en este tipo de respuestas la habituación ocurre meramente por cambios intrínsecos en el circuito reflejo, sin la mediación de influencias extrínsecas asociativas. Ya que tanto la respuesta de sobresalto como la de parpadeo son respuestas de curso temporal muy rápido que ocurren dentro de los primeros $50 \mathrm{~ms}$ de estimulación, es posible conjeturar que en dicho intervalo no se logra el procesamiento del estímulo que se necesita para formar una asociación con el contexto (Jordan et al., 2000; Wagner \& Vogel, 2010).

Wagner y Vogel (2010) esbozaron una explicación cuantitativa de este dilema basada en los modelos SOP (Wagner, 1981) y su extensión AESOP (Wagner \& Brandon, 1989), según la cual las respuestas de procesamiento breve no tendrían la misma facilidad para asociarse con el contexto que las respuestas de procesamiento más largo. Los supuestos son los siguientes:

1) Cada vez que se presenta un estímulo, por ejemplo un tono, se establece una asociación entre el tono, que actuaría como estímulo incondicionado (EI) y el contexto, que actuaría como estímulo condicionado (EC).

2) Según el modelo SOP, tanto el EC como el EI activan un par de unidades de procesamiento: una unidad primaria seguida por una unidad secundaria que recurrentemente inhibe a la primaria. Una vez que se presenta el estímulo correspondiente (contexto o tono) y durante cada momento de su duración, la unidad primaria respectiva es activada, lo cual provoca la respuesta y la activación de la unidad secundaria respectiva. Una vez que es activada, la unidad secundaria recurrentemente inhibe a la primaria, dejándola temporalmente menos susceptible de ser activada por el estímulo. Con el paso del tiempo, la actividad secundaria decae, liberándose a la unidad primaria de la inhibición.

3) El modelo asume que el EC y el EI desarrollan una asociación excitatoria en la medida en que el grado de activación simultánea de sus respectivas unidades primarias es sustancialmente más grande que el grado de activación primaria del EC coincidente con la activación secundaria del EI.

4) Si la asociación que se forma entre el EC (contexto) y el EI (tono) es excitatoria, entonces el contexto adquiere la capacidad, a través de su actividad primaria, para generar asociativamente actividad secundaria en el tono, aun cuando este último no se encuentre presente. Dado que la actividad secundaria del tono inhibe recurrentemente su propia actividad primaria, la presencia del contexto produce una disminución en la respuesta o habituación.

De acuerdo a estos mecanismos, el modelo SOP explicaría la habituación como un fenómeno de primacía. Es decir, a medida que se desarrolla una asociación entre el contexto y el estímulo en cuestión, este último pierde paulatinamente la capacidad para producir su propia respuesta al estar pre-procesado o primado por el contexto. Hasta este 
punto, la predicción del modelo es que la habituación de cualquier sistema de respuesta debería estar controlada por el contexto. Con el fin de explicar el hecho de que el control contextual ocurra con algunos sistemas de respuesta pero no con otros, Wagner y Vogel (2010) apelan a los siguientes supuestos adicionales:

5) Ya que el mismo estímulo incondicionado, por ejemplo un tono, es capaz de generar más de una respuesta, por ejemplo parpadeo y aceleración cardiaca, el modelo debe asumir que la presentación del estímulo provoca la activación de 2 pares de unidades de procesamiento, una para cada respuesta. Esto es consistente con lo planteado por Wagner y Brandon (1989), quienes señalan que los estímulos incondicionados deberían representarse a través de unidades sensoriales y emotivas, donde cada una establecería asociaciones independientes con el estímulo condicionado (contexto). En el presente caso, las unidades sensoriales representarían la respuesta de parpadeo, mientras que las unidades emotivas representarían la respuesta de aceleración cardiaca ante el tono.

6) Se asume que aunque la asociación del contexto con las respuestas sensorial y emotiva sigue las mismas reglas de SOP, hay importantes diferencias paramétricas en el curso temporal de ambas respuestas. Wagner y Vogel (2010) enfatizaron que la diferencia más notable es que el procesamiento emotivo es mucho más lento que el procesamiento sensorial, lo cual le otorga una mayor duración a la actividad primaria emotiva y, por consiguiente, una mayor probabilidad de asociación con el contexto.

De acuerdo a este análisis, es factible esperar que las respuestas de mayor duración muestren una mayor especificidad contextual que las respuestas de corta duración. Si bien esto es congruente con los hallazgos de la presente investigación y con los datos reportados por Jordan et al. (2000), aún quedan incógnitas por resolver, tanto desde el punto de vista teórico como empírico. Desde le punto de vista teórico, el enfoque presentado por Wagner y Vogel (2010) aún requiere de un mayor refinamiento cuantitativo, ya que estos autores tentativamente han concebido al contexto como un estímulo condicionado de larga duración, lo cual representa una sobre-simplificación de su naturaleza multimodal y difusa (p. ej., Fanselow, 2007, 2009; Rudy, 2009). Asimismo, desde el punto de vista empírico se requiere examinar, a través de otros estudios, la existencia de control contextual diferencial de la habituación de otras respuestas con características temporales comparables a aquellas examinadas en el presente estudio. En la medida en que se recolecte información paramétrica acerca de otras respuestas, será posible definir con mayor precisión las condiciones que favorecerían el control contextual de la habituación.

\section{Referencias}

Borszcz, G., Cranney, J., \& Leaton, R. (1989). Influence of long-term sensitization on long-term habituation of the acoustic startle response in rats: Central gray lesions, preexposure, and extinction. Journal of Experimental Psychology: Animal Behavior Processes, 15(1), 54-64.

Castellucci, V., Pinsker, H., Kupfermann, I., \& Kandel, E. (1970). Neuronal mechanisms of habituation and dishabituation of the gill-withdrawal reflex in Aplysia. Science, New Series, 167(3926), 1745-1748.

Davis, M. (1970). Effects of interstimulus interval length and variability on startle-response habituation in the rat. Journal of Comparative and Physiological Psychology, 72(2), 177-192.

Dycus, W., \& Powers, A. (1997). Eyeblink cross-habituation between tactile and acoustic systems in humans. Psychobiology, 25(1), 66-70.

Evans, J., \& Hammond, G. (1983). Differential generalization of habituation across contexts as a function of stimulus significance. Animal Learning $\mathcal{E}$ Behavior, 11(4), 431-434.

Fanselow, M. S. (2007). Context: What's so special about it? En H. L. Roedigger, Y. Dudai \& S. M. Fitzpatrick (Eds.), Science of memory: Concepts (pp. 101-105). Oxford: Oxford University Press.

Fanselow, M. S. (2009). From contextual fear to a dynamic view of memory systems. Trends in Cognitive Sciences, 14(1), 7-15.

Frost, W. N., Brandon, C. L., \& van Zyl, C. (2006). Longterm habituation in the marine mollusk Tritonia Diomedea. Biological Bulletin, 210(3), 230-237. 
Grayson, J. B. (1982). The elicitation and habituation of orienting and defensive responses to phobic imagery and the incremental stimulus intensity effect. Psychophysiology, 19(1), 104-111.

Groves, P., \& Thompson, R. (1970). Habituation: A dual process theory. Psychological Review, 77(5), 419-450.

Hall, G. (1991). Perceptual and associative learning. Oxford: Clarendon.

Hall, G., \& Channell, S. (1985). Differential effects of contextual change on latent inhibition and on the habituation of an orienting response. Journal of Experimental Psychology: Animal Behavior Processes, 11(3), 470-481.

Jordan, W. P., \& Leaton, R. N. (1982). The effects of mesencephalic reticular formation lesions on habituation of the startle and lick suppression responses in the rat. Journal of Comparative and Physiological Psychology, 96,170-183.

Jordan, W., Strasser, H., \& McHale, L. (2000). Contextual control of long-term habituation in rats. Journal of Psychology: Animal Behavior Processes, 26(3), 323-339.

Kruse, A., Stripling, R., \& Clayton, D. (2004). Contextspecific habituation of the zenk gene response to song in adult zebra finches. Neurobiology of Learning and Memory, 82(2), 99-108.

Marlin, N., \& Miller, R. (1981). Associations to contextual stimuli as a determinant of long-term habituation. Journal of Experimental Psychology: Animal Behavior Processes, 7, 313-333.

Peeke, H., \& Veno, A. (1973). Stimulus specificity of habituated aggression in the stickleback (Gasterosteus aculeatus). Behavioral Biology, 8(3), 427-432.

Ponce, F. P., Quintana, G. R., Philominraj, A. S., \& Vogel, E. H. (2011). Habituation of the eyeblink response in humans with stimuli presented in a sequence of incremental intensity. Biological Research, 44(3), 295-299.

Rankin, C. (2000). Context conditioning in habituation in the nematode Caenorhabditis elegans. Behavioral Neuroscience, 114(3), 496-505.

Rudy, J. W. (2009). Context representations, context functions, and the parahippocampal hippocampal system. Learning $\mathcal{E}$ Memory, 16(10), 573-585.
Seal, J. B., \& Zbrozyna, A. W. (1978). Renal vasoconstriction and its habituation in course of repeated auditory stimulation and naturally elicited defense reactions in dogs. Journal of Physiology, 280, 56-57.

Shalter, M., Pentress, J., \& Young, G. (1977). Determinants of response of wolf pups to auditory signals. Behaviour, 60(1-2), 98-114.

Sokolov, E. N. (1960). Neuronal models and the orienting influence. En M. A. Brazier (Ed.), The central nervous system and behavior (pp. 187-276). New York: Macy Foundation.

Thompson, R., \& Spencer, W. (1966). Habituation: A model phenomenon for the study of neuronal substrates of behavior. Psychological Review, 73(1), 16-43.

Tomsic, D., Pedreira, M., Hermitte, G., Romano, A., \& Maldonado, H. (1998). Context-US association as a determinant of long-term habituation in the crab Chasmagnathus. Animal Learning $\mathcal{E}$ Behavior, 26(2), 196-209.

Vogel, E., \& Wagner, A. (2005). Stimulus specificity in the habituation of the startle response in the rat. Physiology E Behavior, 86(4), 516-525.

Wagner, A. R. (1976). Priming in STM: An information-processing mechanism for self-generated or retrieval-generated depression in performance. En T. J. Tighe \& R. N. Leaton (Eds.), Habituation: Perspectives from child development, animal behavior, and neurophysiology (pp. 95-128). Nueva York: Erlbaum.

Wagner, A. R. (1978). Expectancies and the priming of STM. En S. H. Hulse, H. Fowler \& W. K. Honig (Eds.), Cognitive processes in animal behavior (pp. 177-209). Hillsdale, NJ: Erlbaum.

Wagner, A. R. (1979). Habituation and memory. En A. Dickinson \& R. A. Boakes (Eds.), Mechanism of learning and motivation: A memorial volume for Jerzy Konorski (pp. 52-82). Hillsdale, NJ: Erlbaum.

Wagner, A.R. (1981). SOP: A model of automatic memory processing in animal behavior. En N. E. Spear \& R. R. Miller (Eds.), Information processing in animals: Memory mechanisms (pp. 5-47). Hillsdale, NJ: Erlbaum.

Wagner, A. R., \& Brandon, S. E. (1989). Evolution of a structured connectionist model of Pavlovian conditioning (AESOP). En S. B. Klein \& R. R. Mowrer 
(Eds.), Contemporary learning theories: Pavlovian conditioning and the status of traditional learning theories (pp. 149-189). Hillsdale, NJ: Erlbaum.

Wagner, A., \& Vogel, E. (2010). Associative modulation of US processing: Implications for understanding of habituation. En N. Schmajuk (Ed.), Computational models of conditioning (pp. 150-185). Durham: Duke University Medical Center.
Whitlow, J. (1975). Short-term memory in habituation and dishabituation. Journal of Experimental Psychology: Animal Behavior Processes, 1(3), 189-206.

Whitlow, J. W., \& Wagner, A. R. (1984). Memory and habituation. En H. V. S. Peeke \& L. Petrinovich (Eds.), Habituation, sensitization and behavior (pp. 103-153). New York: Academic Press.

Wood, D. C. (1973). Stimulus specific habituation in a protozoan. Physiology $\mathcal{E}$ Behavior, 11(3), 349-354. 